Esta obra está bajo una Licencia Creative Commons Atribución-NoComercial-Compartirlgual 4.0 Internacional

(c) (1) (8) (2)

La seguridad aquí y ahora

Inés Oleastro

DOI: https://doi.org/10.24215/16696581e229

Recibido: 12-06-2019 Aceptado: 15-11-2019

\title{
La seguridad aquí y ahora
}

Reseña del libro "Actores e instituciones de la seguridad en la provincia de Buenos Aires (2010.2018)"

\section{Security here and now \\ Book review "Security actors and institutions in Buenos Aires province (2010.2018)"}

\author{
Inés Oleastro ineoleastro@gmail.com \\ http://orcid.org/0000-0002-8015-0345
}

Instituto de Investigaciones en Humanidades y Ciencias Sociales; Universidad Nacional de La

Plata/ Universidad Nacional de Quilmes/ Consejo Nacional de Investigaciones Científicas y Técnicas (Argentina)

Resumen 
Este trabajo se propone dialogar con los debates sobre la (in)seguridad actuales desde la producción de tesinas de grado de la Licenciatura en Sociología de la Universidad Nacional de La Plata. En este sentido, aborda múltiples problemáticas como la videovigilancia, los dispositivos de control, las políticas de seguridad municipales y la seguridad privada desde la descentralización de la seguridad en estudios etnográficos y cualitativos. Este volumen el primero de una colección más amplia que surge de un proyecto de investigación colectivo sobre el delito en la provincia de Buenos Aires.

Palabras clave: seguridad; dispositivo; delito; municipalización.

\section{Abstract}

This paper dialogue with the debates about security since the production of final degree theses of sociology in the Universidad Nacional de La Plata. In this sense, it addresses multiple problems such as video surveillance, control devices, municipal security policies and private security from the decentralization of security in ethnographic and qualitative studies. This volume is the first of a larger collection that arises from a collective investigation project on crime in the Province of Buenos Aires.

Keywords: security; device; crime; municipalization.

La preocupación por el delito y la (in)seguridad es una dimensión que atraviesa tanto debates académicos como políticos. La evaluación, tratamiento y consecuencias de la (in)seguridad son materia de disputa constante en la escala nacional, provincial y local. Nuevas políticas, dispositivos y respuestas comunales a respecto a las preocupaciones que nos atraviesan como ciudadanos/as e investigadores. Las ciencias sociales se han encargado hace ya un tiempo de comprender y problematizar estas dimensiones. Este volumen, es el primero de una serie de libros reunidos en la colección "Conflicto, violencia y política", que recolecta investigaciones que surgen de tesinas realizadas por estudiantes con el objetivo de finalizar la formación de grado, y que buscan aportar al campo de estudios sobre la seguridad. Además, la colección nace de los debates llevados adelante en el Núcleo de Estudios sobre Seguridad en la provincia de Buenos Aires, como iniciativa de aquel equipo de aportar a través de estas 
investigaciones al campo de estudios de la seguridad, los ilegalismos y el encierro, que veremos también en sus próximas ediciones. Llega además en un momento en el cual la seguridad ha logrado consolidarse como un campo de estudios en sí mismo, y los trabajos alrededor del tema logran avanzar en debates y experiencias empíricas para pensar en nuevos horizontes.

En el caso de este volumen, podemos encontrar plasmada la forma de abordaje de la problemática en la reconstrucción de cada trabajo, en el diálogo con la bibliografía y sobre todo en la introducción del volumen, que nos muestra una amplia revisión de investigaciones sobre la seguridad y reconstruye la perspectiva desde la cual se abordó esta compilación. Cada capítulo de este libro corresponde a una síntesis de cada una de las tesinas que forman parte del volumen. Todas ellas han sido realizadas desde una perspectiva etnográfica, es decir aquella que privilegia la perspectiva del actor/a, la presencia en el campo cotidiana y sostenida por un tiempo determinado por parte del/de la investigador/a. Esta metodología de trabajo es uno de los aportes más importantes de este libro, porque permite abordar los escenarios propuestos en estrecho compromiso con lo que allí sucede. Cada autor/a revive en estas páginas experiencias, discursos y lecturas que permiten adentrarnos en los espacios y los conflictos que allí se intentan mostrar. Gracias a esa inmersión en el campo se puede hacer un aporte peculiar a los estudios sobre seguridad que circulan en las ciencias sociales en general, contribuyendo así con nuevas miradas sobre problemas concretos.

Por otro lado, los trabajos colaboran con análisis sobre la descentralización de las tareas y las respuestas a la inseguridad. Por ello, en cada caso, se abordan experiencias locales de políticas públicas, programas de seguridad o experiencias determinadas alrededor de la prevención del delito. Estos casos se nuclean en la provincia de Buenos Aires pero reconstruyen experiencias locales, lo cual potencia el abordaje etnográfico que previamente destacábamos. En los diferentes trabajos se deja ver la relevancia de pensar al problema público de la inseguridad, las respuestas políticas al respecto y la importancia de la dimensión territorial que de las mismas se desprende. Las investigaciones aquí presentes se concentran entonces en el proceso de municipalización de la seguridad y de profundización de políticas preventivas desde la perspectiva de diferentes actores/as.

Entonces, en este volumen nos encontramos con trabajos que piensan políticas de prevención del delito. Así, Martín Urtasun, reconstruye el dispositivo de video-vigilancia desplegado por el Monitoreo Público Urbano (MoPU) de la ciudad de La Plata, y analiza las vinculaciones entre la política concreta, su implementación y funcionamiento cotidiano y sus relaciones con otras instituciones de control del delito como la policía. Además, deja ver los entramados laborales que existen por detrás de lo que los/as ciudadanos ven, la precarización y la poca calificación 
de su personal. Por último, propone pensar la reproducción de la selectividad y la estigmatización por parte de los/as operadores/as de la video-vigilancia a la hora de poner en práctica el dispositivo. Se destaca aquí una evaluación alrededor del sentido que cobra la supuesta prevención del delito orientada a la localización de sujetos peligrosos para actuar.

Por otra parte, Ana Pasarelli, en su capítulo sobre los corredores seguros de la ciudad de La Plata, cuestiona la incorporación de la demanda vecinal alrededor de la seguridad y la distinción de quiénes representan el peligro. En esta investigación entonces, podemos reparar causas y consecuencias de la problemática de la seguridad con especial hincapié en el tejido social y comunitario. Así, frente a una situación de inconformidad de vecinos de la conocida "zona roja" de la ciudad por la oferta de sexo en el espacio público, así como la presencia de menores de edad que se acercan a comprar y consumir drogas, se llevan adelante una serie de reclamos al municipio. La respuesta, con una clara impronta de intervención en el espacio público, se centra en la creación de un "corredor seguro" en el marco del Sistema Municipal de Prevención y Protección. A partir del despliegue de una serie de visibles mejoras en la zona, los/as vecinos logran concretar su demanda desde una perspectiva de prevención y policiamiento acorde a lo que el paradigma de intervención parece promover.

En lo que respecta a la investigación sobre seguridad privada, Betania Cabandié incorpora un debate interesante sobre el trabajo desplegado por patovicas y controladores. En su regulación estatal y legislativa, se extiende desde el ámbito comercial privado hacia el Control de Admisión y Permanencia de espacios como boliches, bailantas y recitales. Implica entonces un pasaje de lo privado a lo público perforando las fronteras que regularmente separan esos sitios. Lo interesante de este trabajo, que encuentra su potencialidad en la etnografía, es que la autora reconstruye su análisis a partir de distintos paradigmas de intervención que ponen en juego de manera diferencial el uso del cuerpo, la violencia y la regulación de los espacios. Así, nos invita a pensar la seguridad privada en la ciudad de La Plata para dichos eventos desde la implementación de la Ley 26370 que dispara el caso Castellucci, lo que representa una respuesta posible al problema de la inseguridad desde la regulación del trabajo de patovicas y la instalación de un nuevo paradigma, de los controladores/as, que modifique situaciones de violencia y malos tratos en el Control de Admisión y Permanencia.

El trabajo de Belén Roca Pamich, también situado en la ciudad de La Plata, reconstruye diagnósticos y posicionamiento de jóvenes militantes de organizaciones políticas de la Universidad Nacional de La Plata frente al problema de la inseguridad, disparado a partir del robo de bicicletas. La autora propone un análisis comparativo entre dos agrupaciones que representan a nivel nacional partidos políticos históricamente adversarios (peronismo y radicalismo). En este capítulo encontraremos entonces un desafío a las lecturas tradicionales 
sobre la seguridad donde una de las agrupaciones, "Rodolfo Walsh", que es conducción del Centro de estudiantes de la Facultad de Periodismo y Comunicación Social, se corre del sentido común para pensar la inseguridad ya no necesariamente en vinculación con políticas de prevención del delito, ni desde el punitivismo o el policiamiento, sino más bien desde la violencia institucional. La otra organización, la Franja Morada de la Facultad de Ciencias Jurídicas y Sociales, también conducción del Centro de estudiantes, sí se sostiene desde una lectura más tradicional. La misma se sustenta explicaciones en falta de educación o problemas de consumo de drogas. Sin embargo, ambas siguen reproduciendo una conexión instantánea entre seguridad y delito contra la propiedad. Ahora bien, lo interesante que propone Roca Pamich es hacer dialogar en su trabajo estos discursos con el abordaje de problemáticas concretas en los espacio de militancia de aquellos jóvenes que estudia.

Por otro lado, el volumen cuenta con un capítulo destinado al estudio de la policía comunal en una localidad de menos de 70 mil habitantes, de lo que suele referirse como "el interior de la provincia" de Buenos Aires. Este apartado pone en dialogo las reformas diseñadas para la Policía Bonaerense con experiencias concretas de su implementación. Así, Romina Alegre nos introduce a la descentralización de la tarea policial y la puesta en marcha de la policía local y comunal. Sin embargo, en el caso que despliega la autora, podemos conocer los cortocircuitos y las tensiones que se generan entre la intensión de generar una política que incorpore la participación ciudadana con la prevención del delito. Con elementos positivos y negativos en esta transformación para los diferentes actores/as, se pueden ver continuidades con las antiguas policías, así como rupturas o diferencias entre "viejos" y "nuevos" policías. Lo que se muestra, además, es que más allá de la relevancia "del problema de la inseguridad", a nivel local la cuestión se reconfigura de otra manera y los/as funcionarios/as prefieren no hacerse cargo atendiendo a sus consecuencias electorales más inmediatas.

Finalmente, Mercedes Gentile y Vanesa Lio aportan una interesantísima entrevista a la especialista en seguridad Victoria Rangugni. Este cierre es uno de los pilares del volumen, ya que pone en debate teórico y político la seguridad e inseguridad desde la academia y desde la gestión estatal. Tanto en la entrevista como en la introducción propuesta desde la edición, el libro nos introduce y contextualiza sobre los principales debates del campo académico y político sobre la temática a nivel nacional e internacional.

Podemos notar que el libro propone articular distintas experiencias de investigación que si bien son los primeros acercamientos al campo de sus autores implican interesantes aportes al estudio de políticas de seguridad, dispositivos y discursos alrededor del problema de la inseguridad. En este sentido, es a partir de casos concretos y su abordaje etnográfico que logramos pensar cómo se concretan políticas, perspectivas y paradigmas en determinados 
contextos. Lo interesante del volumen es la forma en que estas distintas reconstrucciones ponen en vinculación políticas, demandas y discursos de la seguridad con sus actores/as y territorios concretos.

\section{Bibliografía}

Calandrón, S. y Galar, S. (editores) (2019). Actores e instituciones de la seguridad en la provincia de Buenos Aires (2010-2018). La Plata: Universidad Nacional de La Plata, Facultad de Humanidades y Ciencias de la Educación; CABA: Clacso. Recuperado de https://www.libros.fahce.unlp.edu.ar/index.php/libros/catalog/book/149 